\title{
Displacement: Translation and Rotation. Differences and Similarities in the Discrete and Continuous Models
}

\author{
G. LÁMER \\ University of Debrecen, Faculty of Engineering, Department of Engineering Management and Enterprise, \\ glamer@eng.unideb.hu
}

Abstract. The motion (displacement) of the Euclidean space can be decomposed into translation and rotation. The two kinds of motion of the Euclidean space based on two structures of the Euclidean space: The first one is the topological structure, the second one is the idea of distance. The motion is such a (topological) map, that the distance of any two points remains the same. The bounded and closed domain of the Euclidean space is taken as a model of the rigid body. The bounded and closed domain of the Euclidean space is also taken as a model of the deformable solid body. The map - i.e. the displacement field - of the deformable solid body is continuous, but is not (necessarily) motion; the size and the shape of body can change. The material has atomic-molecular structure. In compliance with it, the material can be comprehended as a discrete system. In this case the elements of the material, as an atom, molecule, grain, can be comprehended as either material point, or rigid body. In the first case the kinematical freedom is the translation, in the latter case the translation and the rotation. In the paper we analyse how the kinematical behaviour of the discrete and continuous mechanical system can be characterise by translation and rotation. In the discrete system the two motions are independent variable. At the same time they characterise the movement of the body different way. For instance homogeneous local translation gives the global translation, but the homogeneous local rotation does not give the global rotation. To realise global rotation in a discrete system on one hand global rotation of the position of the discrete elements, on the other hand homogeneous local rotations of the discrete elements in harmony with global rotation are required. In the continuous system the two kinds of movement cannot be interpreted: a point cannot rotate, a rotation of surrounding of a point or direction can be interpreted. The kinematical characteristics, as the displacement (practically this is equal to translation) of (neighbourhood of) point, the rotation of surrounding of that point and the rotation of a direction went through that point are not independent variables: the translation of a point determines the rotation of the surrounding of that point as well as the rotation of a direction went through that point. With accordance this statement the displacement (practically translation) (field) as the only kinematical variable can be interpreted in the continuous medium.

Key words: displacement, translation, rotation, discrete mechanical model, continuous mechanical model

\section{Introduction}

\section{The subject and aim of research}

To describe the mechanical behaviour of the deformable solid body, the theory of elasticity is used [12]. The kinematical variable in the theory of elasticity is the displacement (practically translation) 
field. In this theory the physical inhomogeneities, internal structure in the material cannot be taken into consideration. To generalize the theory of elasticity used the second kinematical variable, the rotation field (see [4-6]). A point can be shifted, but cannot be rotated. A rigid body with non-zero size can be rotated. The contradictions in the theory of micropolar elasticity and their causes was discussed a few decades ago [8]. Kunin showed, the micropolar theories based on discrete systems [7]. Because of this the solid body with and without structure was analysed [9], as well as the construction of model of discrete and continuous system [11].

The purpose of the study is showing the difference between kinematical behaviour of the discrete and the continuous mechanical systems. At the same time to show the freezing of the internal kinematical variables is that moment which makes to interpret continuous mechanical system possible.

\section{The motions (displacement) of Euclidean space}

The motion (displacement) of the Euclidean space can be decomposed into translation and rotation. The translation as a map is commutative and does not have fix point, the rotation as a map is noncommutative and have a fix point. The two kinds of motion of the Euclidean space based on two structures of the Euclidean space: The first one is the topological structure that fix the order (successiveness) of the points of the space. The second one is the idea of distance. The motion is such a (topological) map that the distance of any two points remains the same [3].

The following relations exist among translation and rotation. The translation is a homogeneous displacement field: the every point of the space has the same translation. The translation can be decomposed into two rotations with the same magnitude but the opposite directions. The rotation is a linearly varying displacement field: the every point of the space moves in a plane orthogonal to the same right line of space (the axe of the rotation), the movement describe a circular arc. The rotation cannot be decomposed into two or any more translations. At the end the any (real) motion of Euclidean space is equivalent to momentary rotation or to translation and rotation acting in the same time. The any (real) motion of Euclidean space can be decomposed into two reflections (non-real motion of Euclidean space) [3].

\section{Deformable solid body, rigid body and material point}

The Euclidean space, more precisely a bounded and closed region of it, is taken as a model of the deformable solid body. In the model one vector-value kinematical degree of freedom, the translation vector, is usually connected to every point of Euclidean space. The map of the solid body - the translation field - is continuous, but it is not necessarily motion; the size and the shape of the body can change.

The rigid body is such a model of the solid body in which the body does not change its size. More precisely, the distance between any two points of the body remains the same under map. That is the rigid body is regarded as a bounded and closed region of Euclidean space with condition, that the distance between any two points of the region remains the same under map. 
The (real) motion of the rigid body is translation and rotation, see Fig. 1. That is the kinematical degree of freedom of rigid body are translation vector and rotation vector (which objects are connected to the reference point of the rigid body).

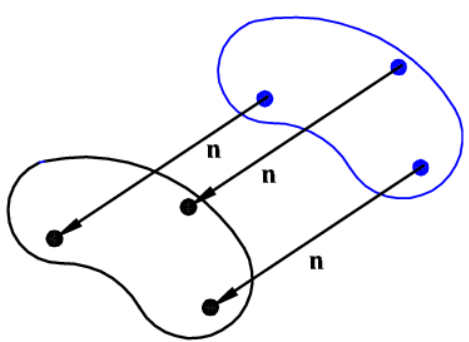

a) Translation

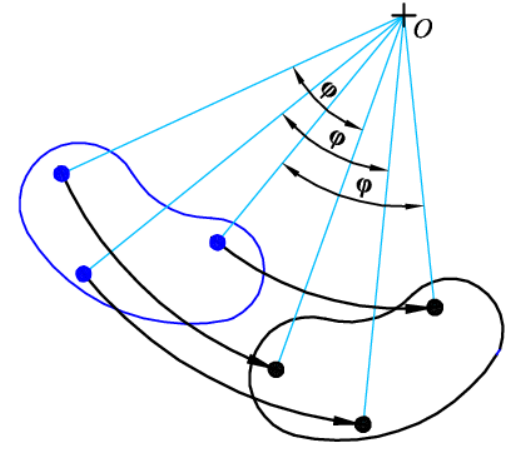

b) Rotation

Figure 1. Rigid body like motion of rigid body: translation and rotation.

The material point is regarded as a bounded and closed region of Euclidean space with condition, that under map only one point, the reference point of body is played attention to. The actual position of the points of body are not examined. So we can suppose the body is small, but this assumption has no meaning because of this assumption we simple not use: only the position of one reference point of the body figures in the model.

The (only) motion of the material point is translation. That is the kinematical degree of freedom of material point is translation vector (which object is connected to the reference point of the material point).

\section{Deformable solid body}

The material has atomic-molecular structure. In accordance with this the material can be regarded as discrete system. In this case the particle - i.e. atoms, molecules, grains - that built the material can be regarded as material point or rigid body. In the first case the kinematical degree of freedom is translation vector, in the latest case the kinematical degrees of freedom are translation vector and rotation vector.

The concrete is regarded as a deformable solid body. The concrete is composite material: consist of pebble, sand and cement-stone. The sand-cement-stone is usually called matrix. On the sectionsurface of concrete the pebble and the sand-cement-stone are separated well. In the deformation of concrete both pebble and sand-concrete-stone change the shape and the size (see Fig. 2.).

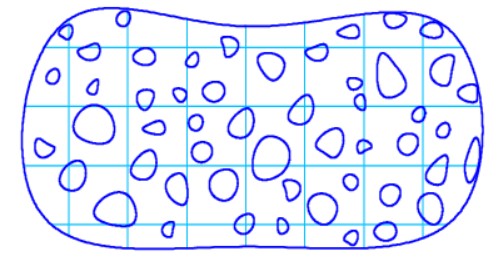

a) Undeformed state

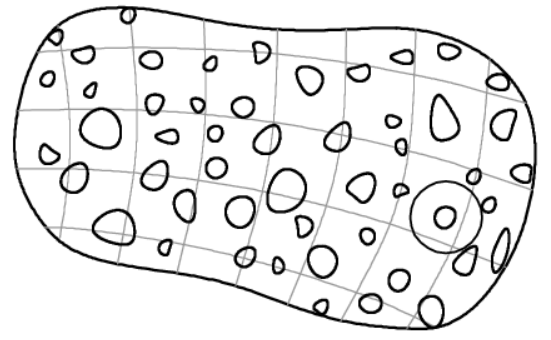

b) Deformed state

Figure 2. Representation of deformation of the deformable solid body (concrete) showing the internal structure 106 
In the analysis of body the inhomogeneity (internal structure that is pebble and sand-cement-stone) is usually disregarded, consequently the deformations of body are described by bending and elongation of coordinate lines taken up inside the body (see Fig. 3.).

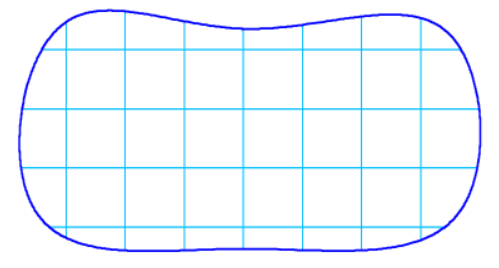

a) Undeformed state

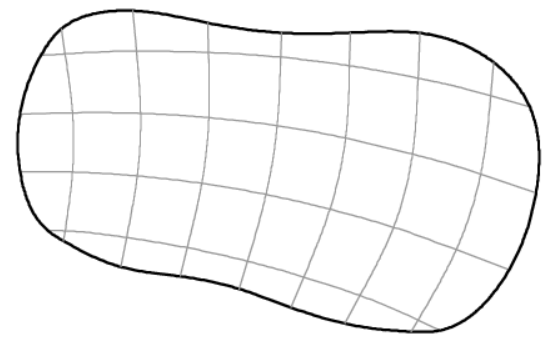

b) Deformed state

Figure 3. Representation of deformation of the deformable solid body showing the deformation of coordinate lines

The deformable solid body is solid that under deformation does not fall into pieces. That is the picture shown in Fig. 4. does not refer to the deformable solid body but to body built from cube sugars.

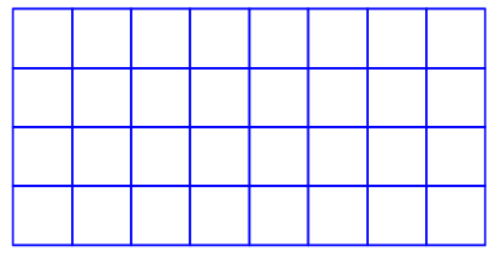

a) Undeformed state

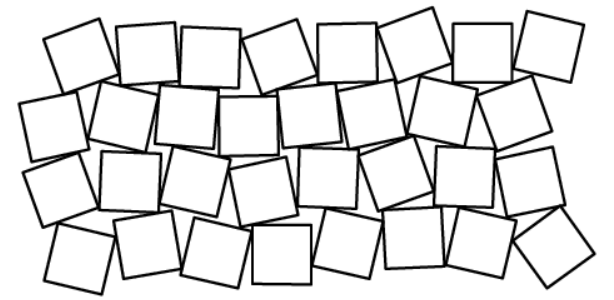

b) Deformed state

Figure 4. Change of state of body built from cube sugars

At the same time that question is arisen, whether how can, or how must imagine the deformable solid body built up from elementary particles, that is material points or rigid bodies. The model consisting of material points sitting in the node of lattice is following (see Fig. 5.).

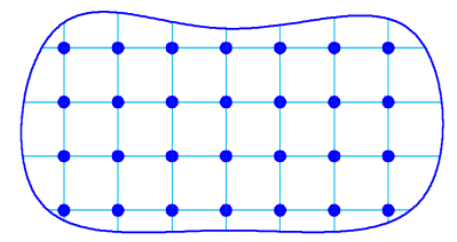

a) Undeformed state

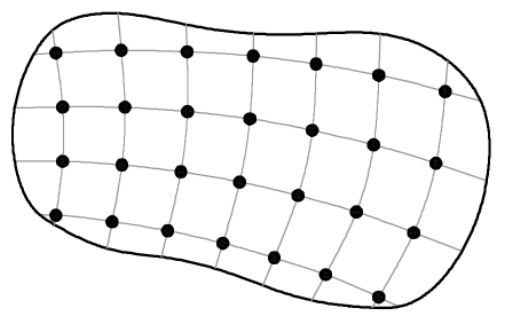

b) Deformed state

Figure 5. The model consisting of material point sitting in the node of lattice

Let the deformable solid body built up from rigid bodies!

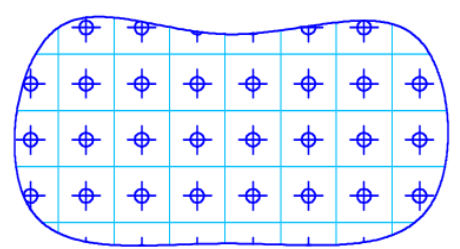

a) Starting state

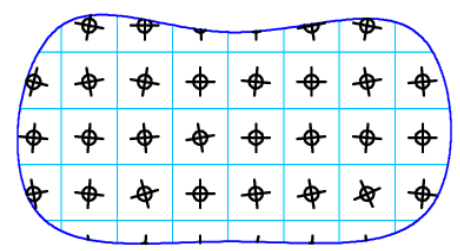

b) Rotation only rigid bodies

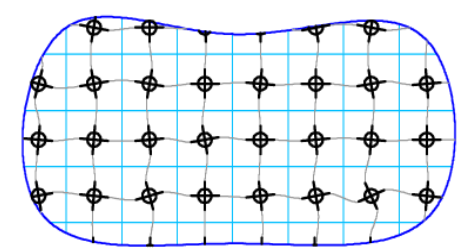

c) Deformation of network lines connecting the rigid bodies

Figure 6. Model of deformable solid body built up from rigid bodies I.: only local rotations 
The lines which designates the cell will be named as coordinate lines (see Fig. 6.a), the line which connect rigid bodies will be named as network lines (see Fig. 6.c). In the case of solid body built up from rigid bodies the shape of the body not need necessarily change when only the rigid bodies rotate, but the network lines connecting the rigid bodies become deformed (see Fig. 6.).

Although, under translations and rotations of rigid bodies the shape of the solid body changes, but the deformation of network lines connecting rigid body differs from deformation of coordinate lines because of independence of translation from rotation of rigid bodies (see Fig. 7.a). Though the deformations of two nets of lines can be harmonized, the independence from each other rotations of every rigid body remains (see Fig. 7.b). Of two kinds rigid body like motions (translation and rotation) cannot be connected (it is assumed that the network lines can be approximated by coordinate lines) because of the moving trihedron of coordinate lines cannot be covered by rigid body's cross axes: the intersected coordinate lines not only change own form (they are bent) but the angle between them also changed (see Fig. 7.c).

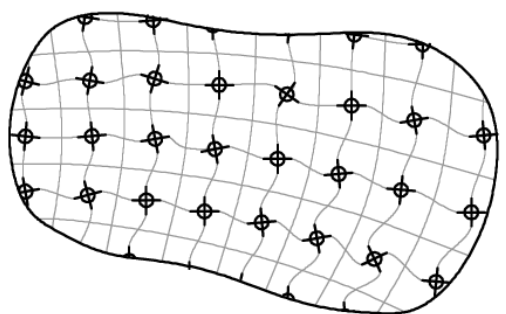

a) Deformation of network lines connecting rigid bodies

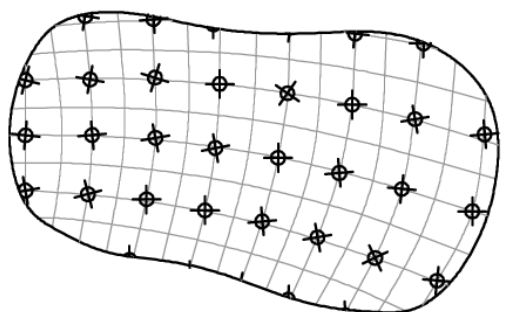

b) Correcting deformation of network lines connecting rigid bodies

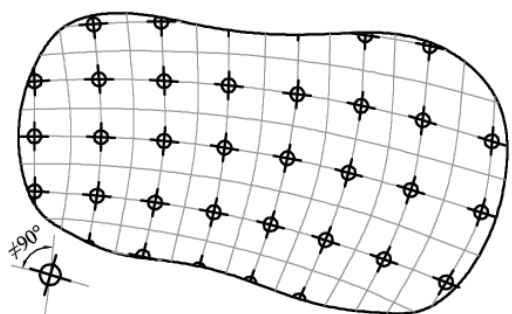

c) Deformation of continuous solid body

Figure 7. Model of deformable solid body built up from rigid bodies II.: the continuous translation field but different local rotations
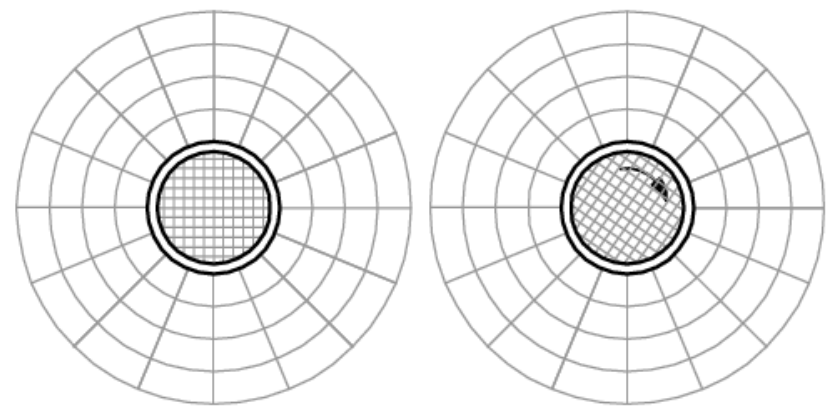

a) The deformation of matrix and the rotation of rigid body are independent
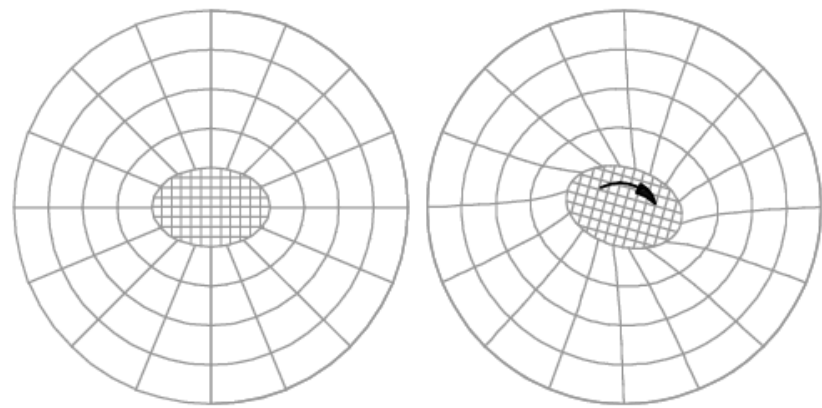

b) The deformation of matrix and the rotation of rigid body are connected

Figure 8. The embedding of rigid body in a matrix 
The embedding of rigid body in a matrix can be of two kinds. (See the marked with a circle "pebble" on the left side of the Fig. 2.b!) The first, the embedded rigid body can rotate independently from the matrix as a ball in the bearing (Fig. 8.a). The second, that embedded rigid body does not able to rotate independently form matrix, the two elements are connected (Fig. 8.b).

The presented figures draw attentions to the facts that the models of deformable solid body built up from material points or rigid bodies isn't in accordance with lifelike deformations of the deformable solid bodies.

\section{Flowing body}

The flowing body is such a body in which the particles (atoms, molecules, grains) do not fixed each to other: though particles are in connections with other ones but their order continuously changes. Consequently the continuous map of a region of Euclidean space dos not reflect just the continuous changes of positions of particles.

The continuous model contains other contradictions too. E.g. the vortex motion, the slipping of layers each on other, the stream in varying cross sections are described by continuous functions, but it is evident that these movements of flowing body cannot described by continuous map of a region of Euclidean space. Two pictures are selected from well-known reference (see Fig. 9. in [15] p. 360., as well as Fig. 10.a and Fig. 10.b in [15] p. 362.). Fig. 9. can represent continuous translation in that case when the layers move parallel each with other. (In reality the translation field does not continuous in later case, because of the layers slip each on other. (To go into detail pass beyond the aim of our study.)

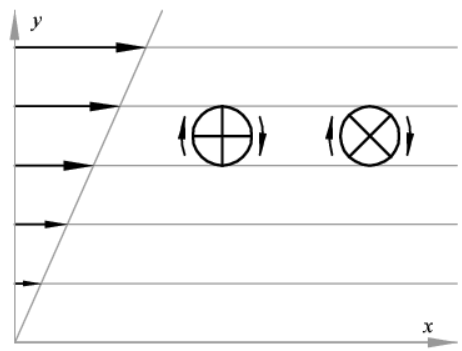

Figure 9. Laminar stream of liquid with inner rotation

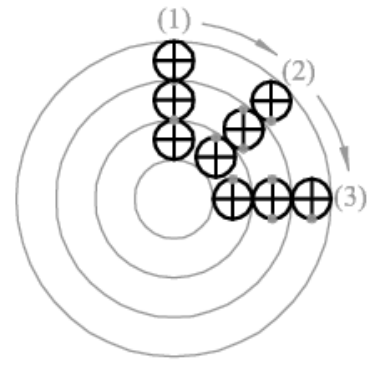

a) Vortex motion with displaced rigid body like particle

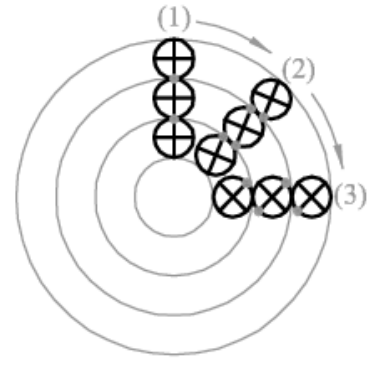

b) Vortex motion with independently displaced and rotated rigid body like particle

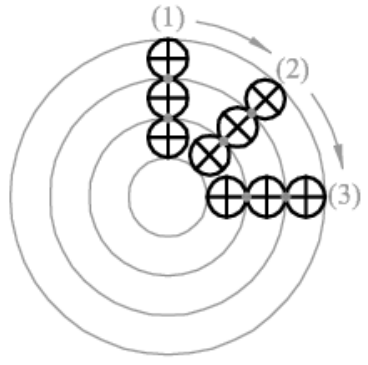

c) Vortex motion with harmonically displaced and rotated rigid body like particle

Figure 10. Vortex motion of liquid with and without inner rotation 
The Fig. 10.a and Fig. 10.b do not represent continuous translation field (we completed the figures to threexthree circle), see the ends of orthogonal straight-line segments. The continuous translation field is given on Fig. 10.c. In this case the elementary particles of medium are not simply displaced and rotated (so the two kinds of motion are not independent), but those are in harmonically displaced and rotated (so the two kinds of motion are harmonized). So a continuous translation field is formed, what represents the rotation of the whole space (plane).

Two another pictures are selected from other well-known reference (see Fig. 11. in [13] p. 43., and p. 205. The continuous map gives continuous streamlines in every cross section of stream, but particles of liquid can flow through varying cross section with change of places because of incompressibility of elements of liquid. So the streamlines of liquid must cross each other. The requirement of incompressibility can be interpreted if the motion, that is the change in time, is taken into consideration: the velocity of particle of liquid changes in the changed cross section. At the same time this statement does not mean that the streamline of liquid would be continuous.
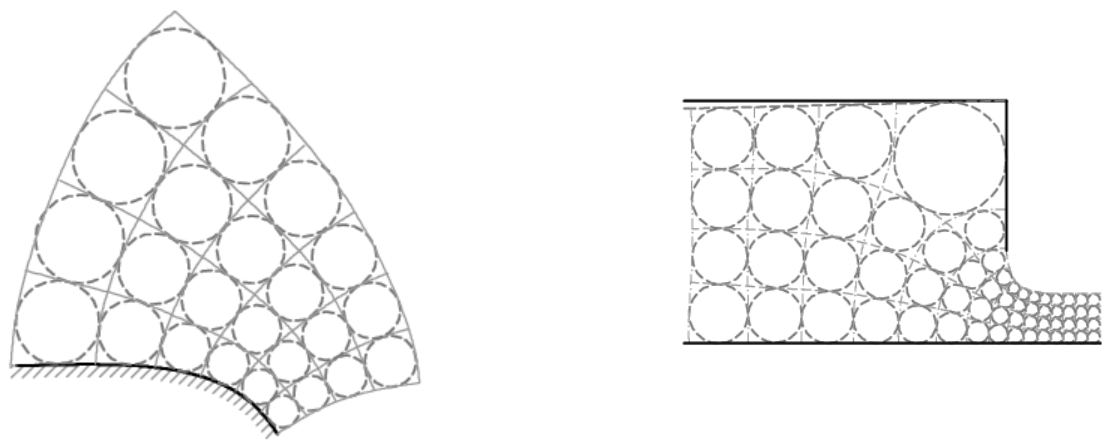

Figure 11. Changed cross section and harmony of continuous map

Note. These phenomena call attentions to the facts that the solid body is continuous in different manner as the flowing body. A hole can be drilled in the solid body and a solid body cannot move in another one. A hole cannot be drilled in the flowing body and a solid body can move in flowing body. While the two bodies are regarded as continuous body from the everyday point of view (see [9]).

The presented figures draw attentions to the facts that the continuous models of flowing body isn't in accordance with physical behaviour (that is with phenomenon of flowing) of liquid.

In the present study the kinematical problems of discrete and continuous models of solely deformable solid body (built up from material points or rigid bodies) are discussed.

\section{Discrete system consisting of material points}

\subsection{The interpretation of the discrete system consisting of material points}

The discrete system consisting of material points is a set of material points sitting in the nodes of the regular network (as a system of crystal lattice) (see Fig. 12.a). The kinematical degree of freedom of material point is the translation. The material point can move from node of the network, but they cannot change own position, more precisely they cannot be rearranged. In other formulation, the network can be deformed, but the material points cannot change own topological order. 
The "sitting in the nodes of the regular network" will be marked by SNRN in the naming of the figures.

\subsection{Disordered displacement (translation) field: the thermal motion}

The state without movement is the basic state. The disordered movement around nodes is the thermal motion (see Fig. 12.b).

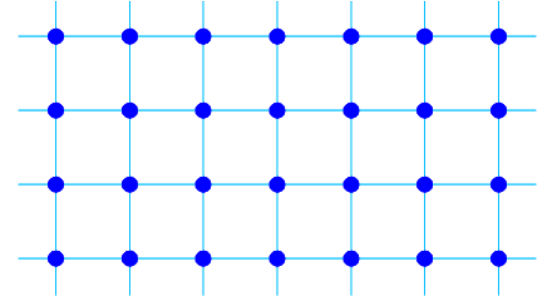

a) The system of points

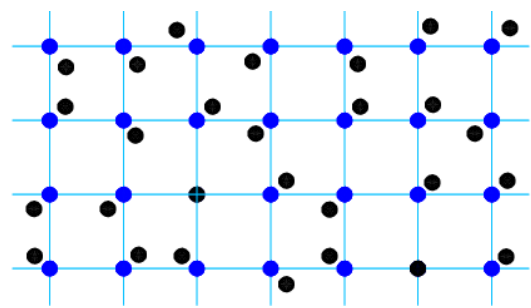

b) Disordered translation field

Figure 12. The system of material points SNRN and the disordered translation field

\subsection{Ordered displacement (translation) field}

Homogeneous local translation: global rigid body like translation.

Rotation around a point: global rigid body like rotation.

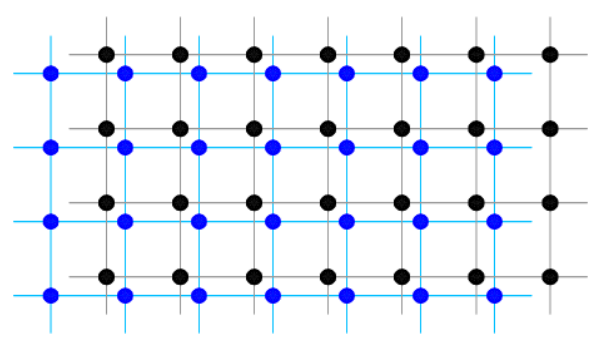

a) Translation

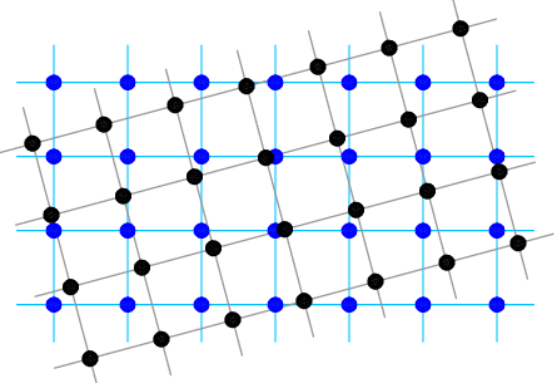

b) Rotation

Figure 13. The global rigid body like translation and rotation of the system of material points SNRN

Linearly varying magnitude of translation in direction of translation vector, or in direction orthogonal to translation vector: homogeneous elongation and homogeneous (simple) shear, respectively.

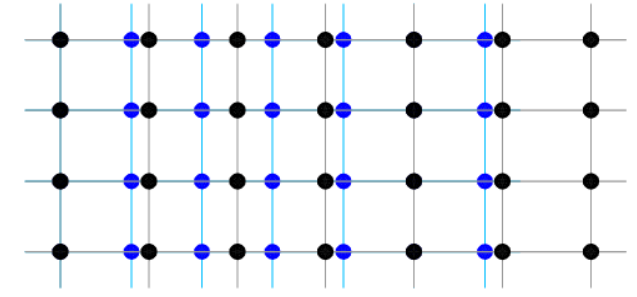

a) Homogeneous elongation

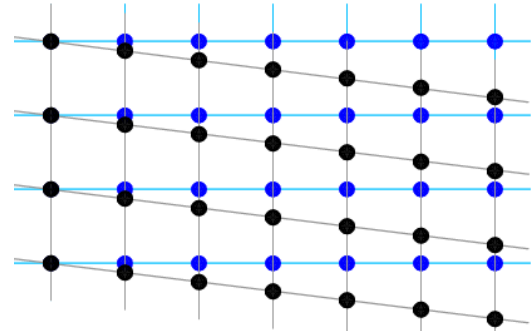

b) Homogeneous (simple) shear

Figure 14. Homogeneous elongation and homogeneous (simple) shear of the system of material points SNRN

Quadratically varying magnitude of translation in direction of translation vector, or in direction orthogonal to translation vector: linearly varying elongation and linearly varying (simple) shear, respectively. 


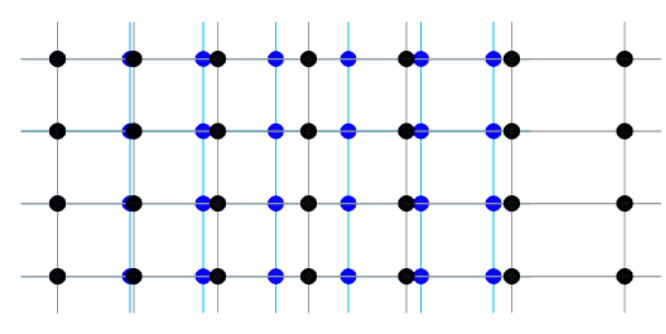

a) Linearly varying elongation

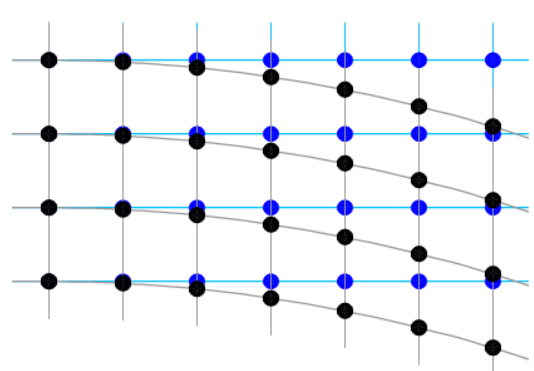

b) Linearly varying (simple) shear

Figure 15. Linearly varying elongation and linearly varying (simple) shear of the system of material points SNRN

The material points sitting on the favoured lines move in the direction orthogonal to the favoured lines according to quadratic law and move in the direction of the favoured lines keeping (according to the one of the geometrical hypotheses of rod's and shell's theory) orthogonality to the favoured line: pure bending.

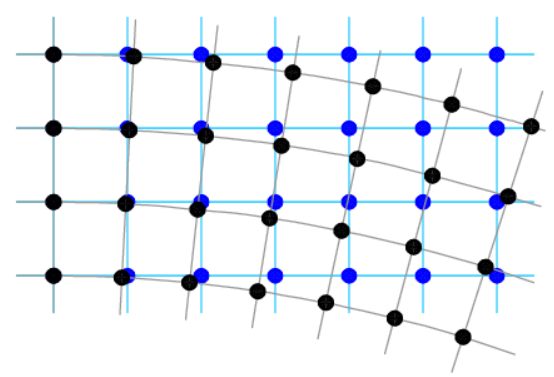

Figure 16. Translation in the direction orthogonal to favoured lines according to quadratic law and in the direction of the favoured lines keeping orthogonality to the favoured line: pure bending of the system of material points SNRN

Notes. 1. The points have no directions, so the ordered motion of the system of material points is analogous to the ordered motion of continuous model (see Chapter 4.) 2. To interpret pure bending the existence of geometrical hypothesis is necessarily and sufficient because of the rotation of material point does not interpreted.

\subsection{Continuous and non-continuous movement}

The movement (translation) of the body consisting of material points can be continuous, or noncontinuous, that is discrete. The movement is continuous, if material points shift collective (see i.e. Fig. 17.). If this movement is regarded as an oscillatory one than this continuous oscillatory movement is usually named acoustic vibrations of crystal lattice.

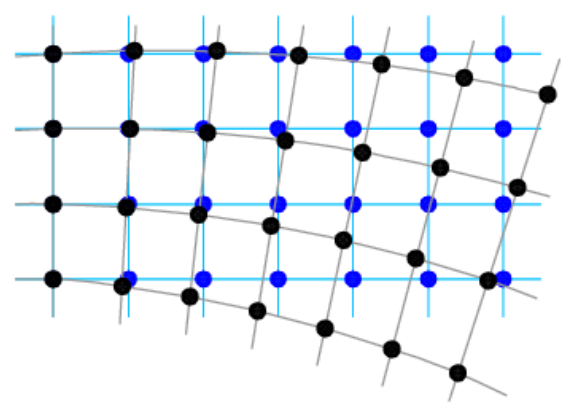

Figure 17. Continuous translation of the system of material points SNRN 
The movement is discrete, if material points shift non-collective but they move independently each from other. Trivial example is the thermal motion, see Fig. 12.b). Discrete movement can be given by different "patterned" movements. Let we take material points arranged in more rows (see Fig. 12.b). The material points under and above each other form a column. That movement of the system of material point is discrete in which every material point in every first column moves right, every material point in every second column moves left (see Fig. 18.), or every material point in every first column moves right, every material point in every third column moves left, and every material point in every second and fourth columns stay on its place (that is these material points do not move) (see Fig. 19.).

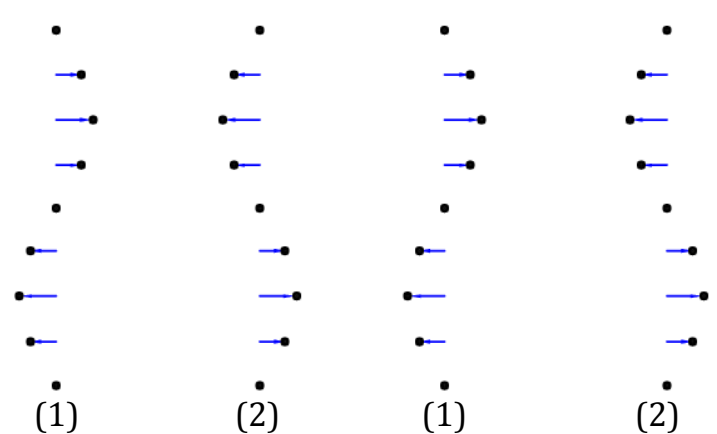

Figure 18. Discrete translation of material points periodically arranged on a straight line I.: two-phase translation

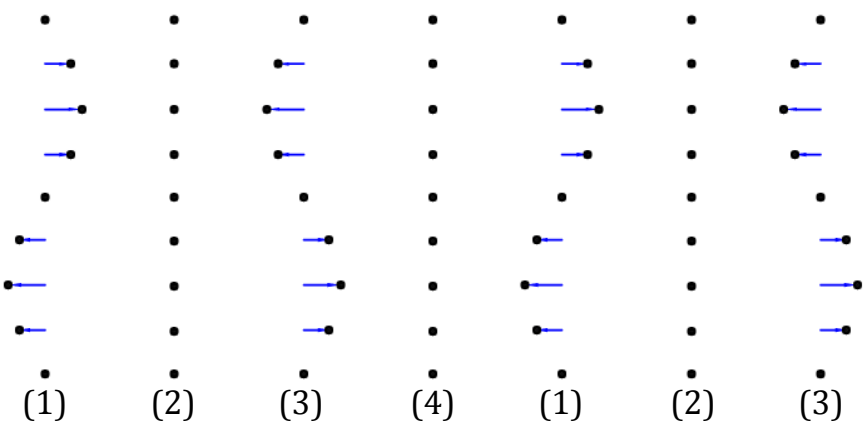

Figure 19. Discrete translation of material points periodically arranged on a straight line II.: three-phase translation

On the Fig. 18. and 19., we represent the discrete movement of the material points as an oscillatory one, and a whole period is presented by eight phases. This discrete oscillatory movement is usually named optical vibrations of crystal lattice.

\section{Discrete system consisting of rigid bodies}

\subsection{The interpretation of the discrete system consisting of rigid bodies}

The discrete system consisting of rigid bodies is a set of rigid bodies sitting in the nodes of the regular network (as a system of crystal lattice) (see Fig. 20.). The grid-mesh of lattice, or skeleton will be used as a synonym of regular network. The kinematical degree of freedom of rigid body are the translation and rotation. The rigid body can move from node of the network, but they cannot change own position. At the same time the rigid body can rotate in the node or in the displaced position. That is the network of the discrete system consisting of rigid bodies can be deformed, but the rigid bodies cannot change 
own topological order. The deformation of the grid-mesh is determined by translations of rigid bodies, at most it will be corrected by rotation of rigid bodies.

The "sitting in the nodes of the regular network" will be marked by SNRN in this section too.

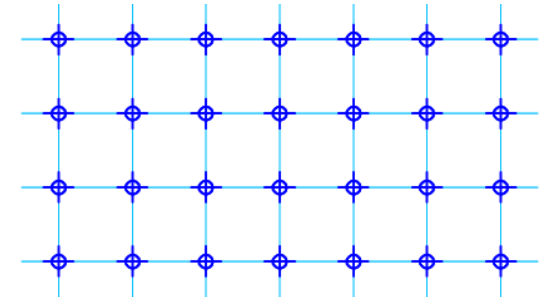

Figure 20. The system of rigid bodies SNRN

\subsection{Disordered displacement (translation and rotation) field: the thermal motion}

The state without movement is the basic state (see Fig. 20.). The disordered translation and rotation around nodes is the thermal motion (see Fig. 21.). Accordingly to the two kinds of movement there are two kinds of thermal motion.

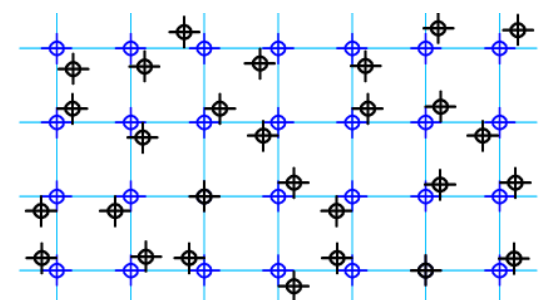

a) Disordered translation field

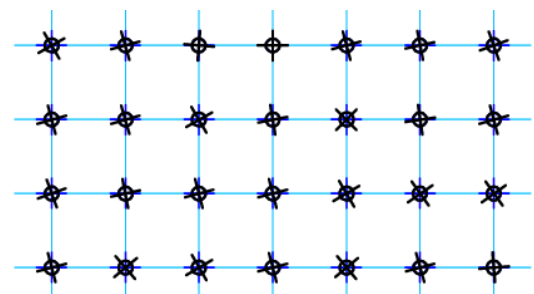

b) Disordered rotation field

Figure 21. The system of rigid bodies SNRN and the two kinds of disordered movements

\subsection{Ordered displacement field (translation and rotation)}

Homogeneous local translation: global rigid body like translation (the shift of rigid bodies takes the grid-mesh of lattice). The duality of this: global rigid body like translation of the grid-mesh of the lattice, local movement does not exist. This movement generate the global rigid body like translation of the whole system (the shift of the grid-mesh of lattice takes the rigid bodies) (see Fig. 22.a).

Homogeneous local rotation: this is the state itself (Fig. 22.b), it is not equal to global rotation, because of the grid-mesh of lattice is motionless.

The global rigid body rotation of the grid-mesh of lattice, there is no local rotation: this is the state itself (Fig. 22.c), it is not equal to global rotation, because of the rigid bodies are rotationless.

The global rigid body rotation of the grid-mesh of lattice + homogeneous local rotation: this is the state itself. (The general case does not shown in Fig. 22., two special cases are given, there is no homogeneous rotation (Fig. 22.c) and the magnitude of the homogeneous local rotation equal to the magnitude of rotation of the grid-mesh of lattice (Fig. 22.d).) 
The global rigid body rotation of the grid-mesh of lattice + homogeneous local rotation with the same magnitude and direction of global rigid body rotation: global rotation (see Fig. 22.d).

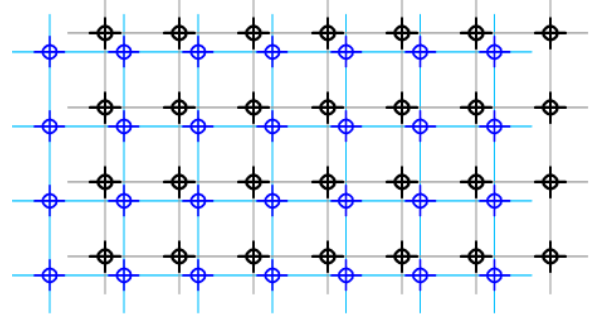

a) Homogeneous local translation = = global translation

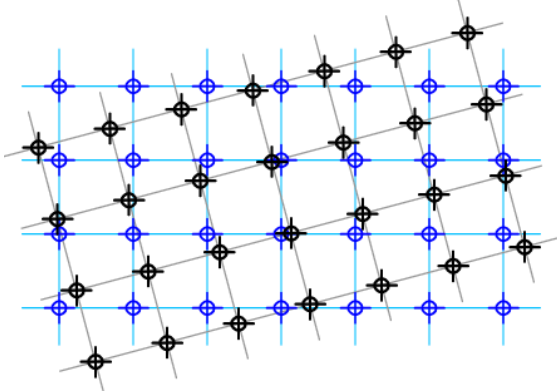

c) The global rotation of skeleton, there is no local rotation

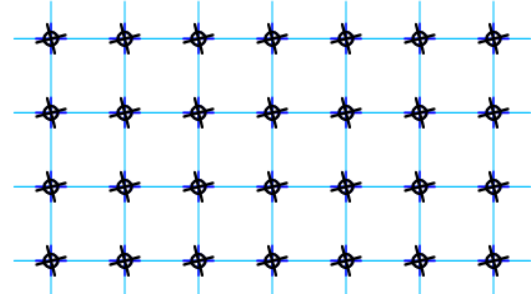

b) Homogeneous local rotation

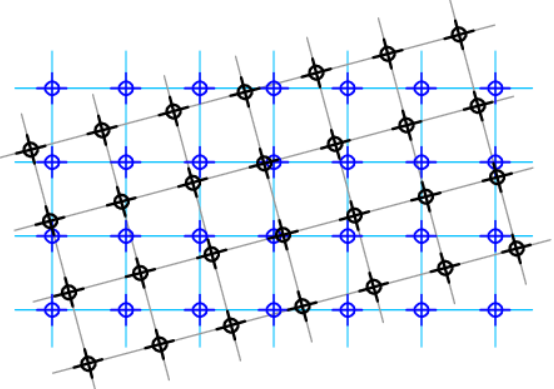

d) The global rotation of skeleton and the homogenous local rotation with the same rotation $=$ global rotation

Figure 22. The system of rigid bodies SNRN and the local and global translations and rotations and their combinations

The rigid bodies have homogeneous, linearly and quadratically varying in magnitude translation in direction of translation vector, there is no local rotation (see Fig. 23.). The homogeneous local translation gives global translation, the linearly varying magnitude of local translation gives homogeneous local elongation, and the quadratically varying magnitude of local translation gives linearly varying local elongation.

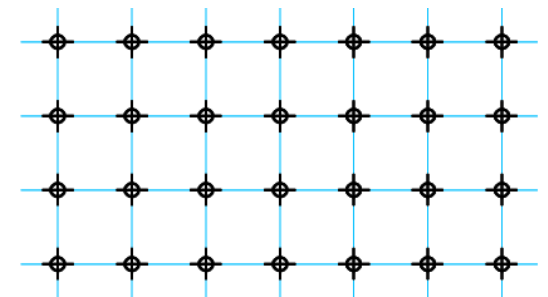

a) The beginning state

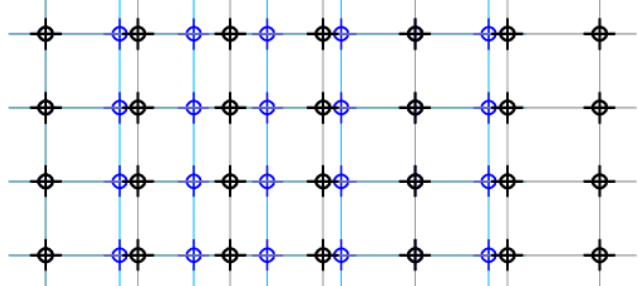

c) Homogeneous elongation

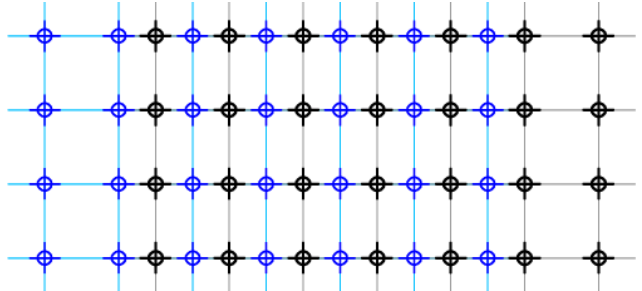

b) Global translation

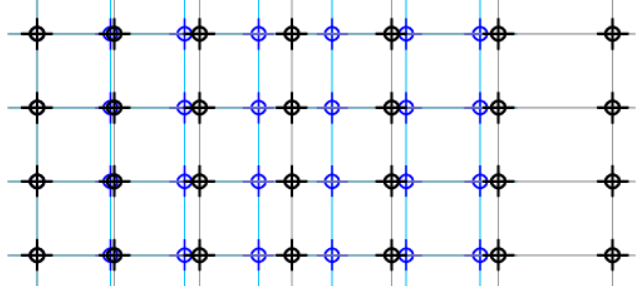

d) Linearly varying elongation

Figure 23. The system of rigid bodies SNRN and the homogeneous, linearly and quadratically varying in magnitude translation in the direction of the translation, there is no local rotation 
The rigid bodies have null, homogeneous, linearly and quadratically varying rotation in a given direction, there is no local translation (see Fig. 24.). These states cannot interpreted in the case of deformable solid body.

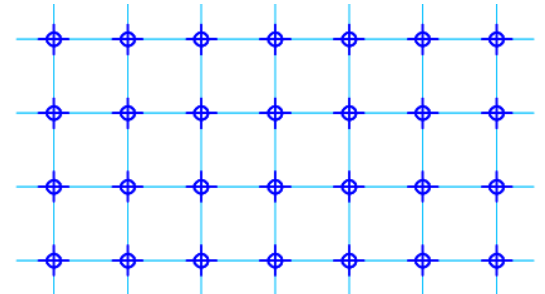

a) There is no local rotation

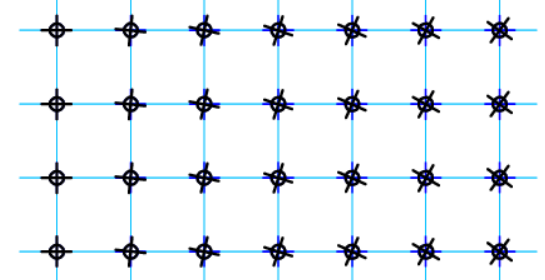

c) Linearly varying local rotation

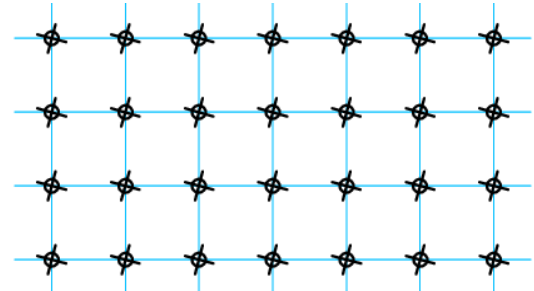

b) Homogeneous local rotation

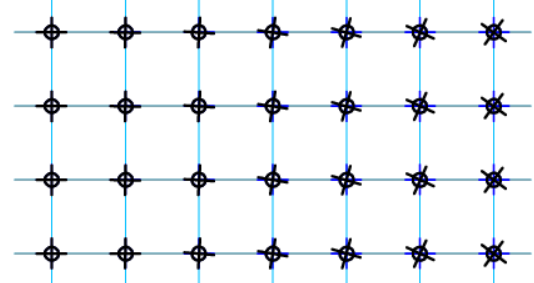

d) Quadratically varying local rotation

Figure 24. The system of rigid bodies SNRN and the null, homogeneous, linearly and quadratically varying rotation in a given direction, there is no local translation

The rigid bodies have linearly varying in magnitude translation in direction orthogonal to translation vector (like the simple shear translation), with null, homogeneous, linearly and quadratically varying local rotation (see Fig. 25.). These states cannot interpreted in the case of deformable solid body.

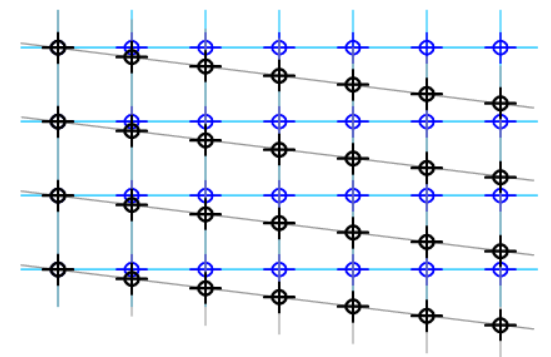

a) There is no local rotation

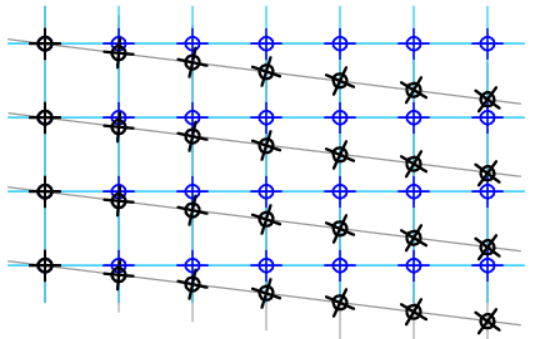

c) Linearly varying local rotation

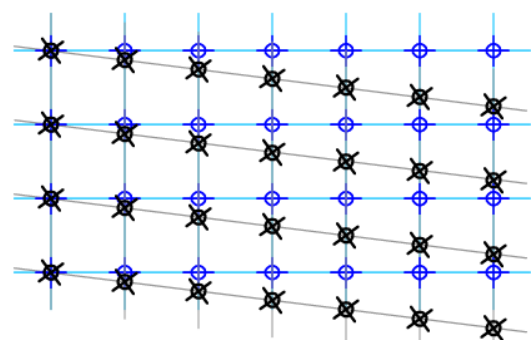

b) Homogeneous local rotation

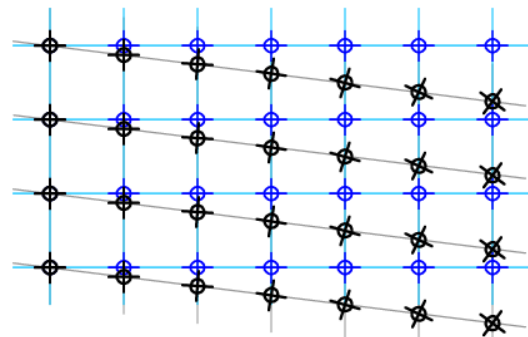

d) Quadratically varying local rotation

Figure 25. The system of rigid bodies SNRN and the null, homogeneous, linearly and quadratically varying rotation in a given direction, there is a homogeneous simple shear-like local translation

The rigid bodies have quadratically varying in magnitude translation in direction orthogonal to translation vector (like the simple shear translation), with null, homogeneous, linearly and quadratically varying local rotation (see Fig. 26.). These states cannot interpreted in the case of deformable solid body. 


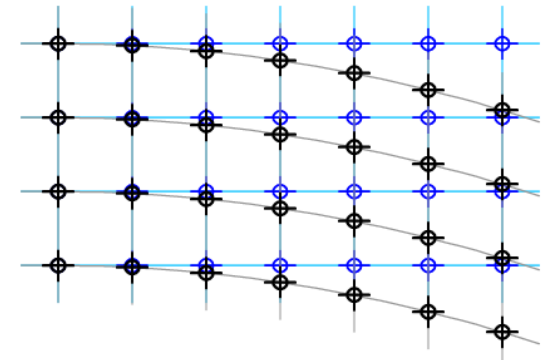

a) There is no local rotation

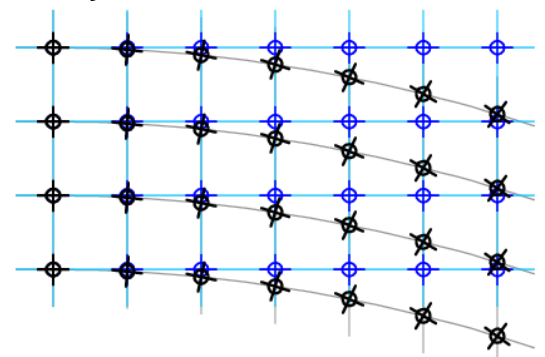

c) Linearly varying local rotation

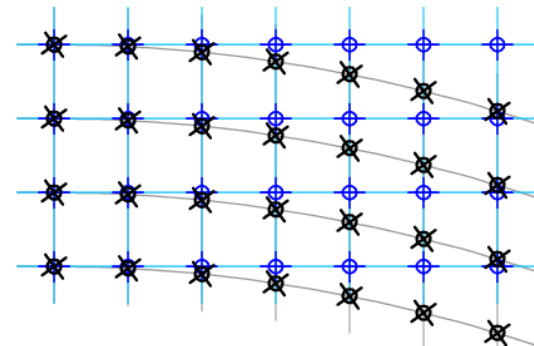

b) Homogeneous local rotation

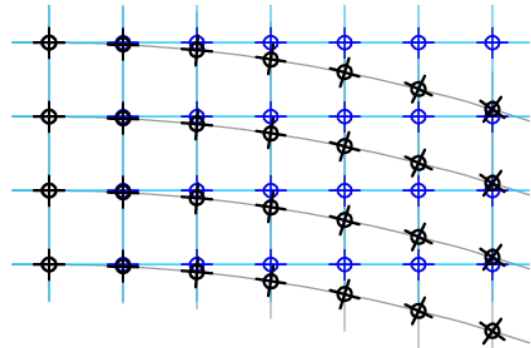

d) Quadratically varying local rotation

Figure 26. The system of rigid bodies SNRN and the null, homogeneous, linearly and quadratically varying rotation in a given direction, there is a linearly varying simple shear-like local translation

The rigid bodies sitting on the favoured lines move in the direction orthogonal to favoured lines according to quadratic law and move in the direction of the favoured lines keeping (according to the one of the geometrical hypotheses of rod's and shell's theory) orthogonality to the favoured line; at the same time the rigid bodies rotate null, homogeneous, linearly and quadratically varying in the same direction. The change of shape of the skeleton given by rigid bodies corresponds to pure bending, but these states cannot interpreted in the case of deformable solid body.

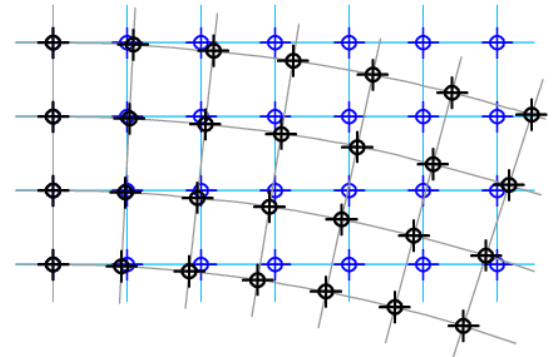

a) There is no local rotation

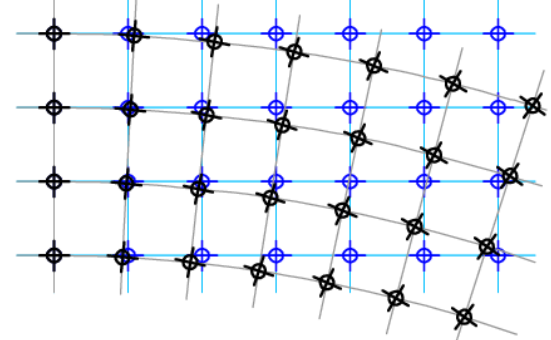

c) Linearly varying local rotation

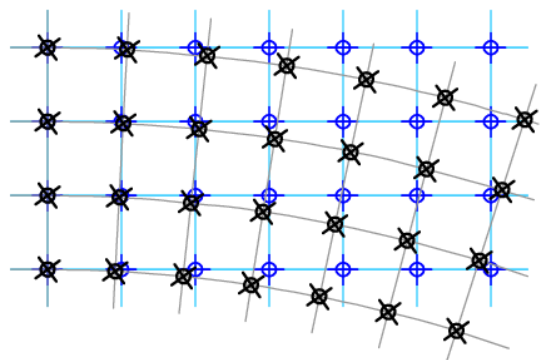

b) Homogeneous local rotation

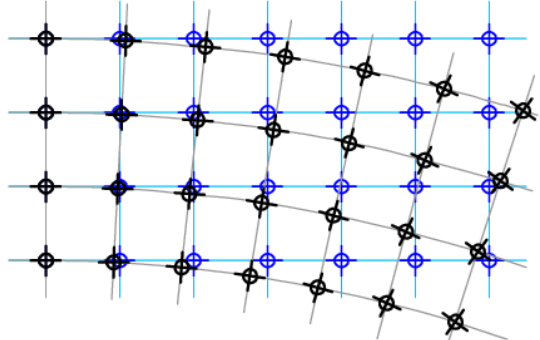

d) Quadratically varying local rotation

Figure 27. Translation in the direction orthogonal to favoured lines according to quadratic law and in the direction of the favoured lines keeping orthogonality to the favoured line: pure bending of the skeleton of the system of rigid bodies SNRN with null, homogeneous, linearly and quadratically varying rotation in a given direction 
It can be read from Fig. 27., the geometrical hypotheses of rod's and shell's theory does not sufficient that the model of deformable solid body could be built form rigid bodies arranged periodically. To do it an additional condition (hypothesis) is necessary: the rigid bodies must rotate so as the moving trihedrons of quadratically bending lines rotate (see Fig. 28.b). This calls attention to fact that quadratically varying translation in cross direction cannot interpreted as simple shear, surely the translations in longitudinal and cross directions must be brought in harmony in such a way, the rigid bodies occupied place on one straight line before deformation must take place on a circle after deformation and the rigid bodies occupied place on an orthogonal straight line before deformation must take place also on orthogonal straight line after deformation (see Fig. 28.).

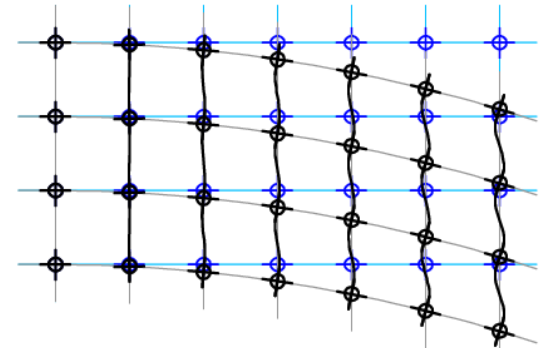

a) Without translation in direction of favoured line

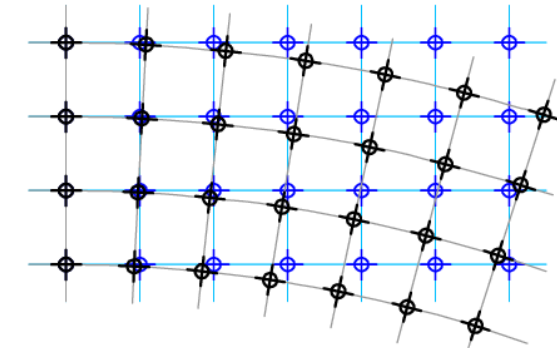

b) Translation in direction of favoured line corresponds to the geometrical hypothesis

Figure 28. In the system of rigid bodies SNRN the rigid bodies rotated in moving trihedrons quadratically varying in magnitude translation in direction orthogonal to favoured line. a) Without translation in direction of favoured line.

b) Translation in direction of favoured line corresponds to the geometrical hypothesis

\subsection{Continuous and non-continuous movement}

Both translation and rotation of the rigid bodies consisting the deformable solid bodies can be continuous, or non-continuous, that is discrete. If this movement is regarded as an oscillatory one than this continuous oscillatory movement (see Fig. 29.) is usually named acoustic vibrations of crystal lattice; but in the case of solid body built up from rigid bodies there are two kinds of acoustic vibrations: vibration of translation and rotations.

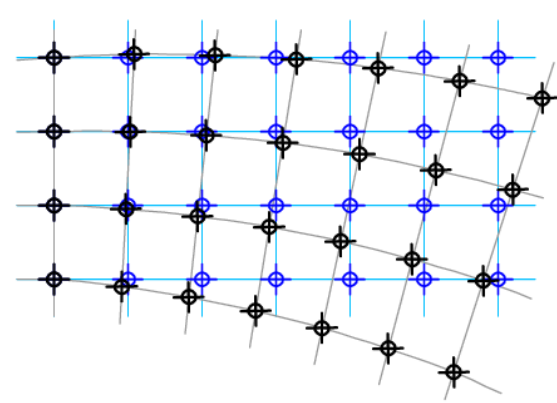

a) Continuous translation

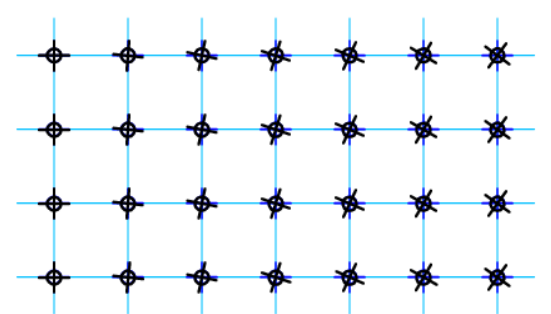

b) Continuous inner rotation

Figure 29. Continuous translation of the system of rigid bodies SNRN

The movement is discrete, if rigid bodies shift or rotate non-collective but they move independently each from other. Trivial example is the thermal motion, see Fig. 21.); accordingly to the two kinds of movement there are two kinds of thermal motion. Discrete movement can be given by different "patterned" movements. Let we take rigid bodies arranged in more rows (see Fig. 20.). The rigid 
bodies under and above each other form a column. That movement of the system of rigid bodies is discrete in which every rigid body in every first column moves or rotates right, every rigid body in every second column moves or rotates left (see Fig. 30.), or every rigid body in every first column moves or rotates right, every rigid body in every third column moves or rotates left, and every rigid body in every second and fourth columns stay on it place (that is these rigid bodies do not move and do not rotate) (see Fig. 31.). On the Fig. 30. and 31., we represent the discrete movement of the rigid bodies as a oscillatory one, and a whole period is presented by eight phases. This discrete oscillatory movement is usually named optical vibrations of crystal lattice; accordingly to the two kinds of movement there are two kinds of optical vibrations, and its combinations.

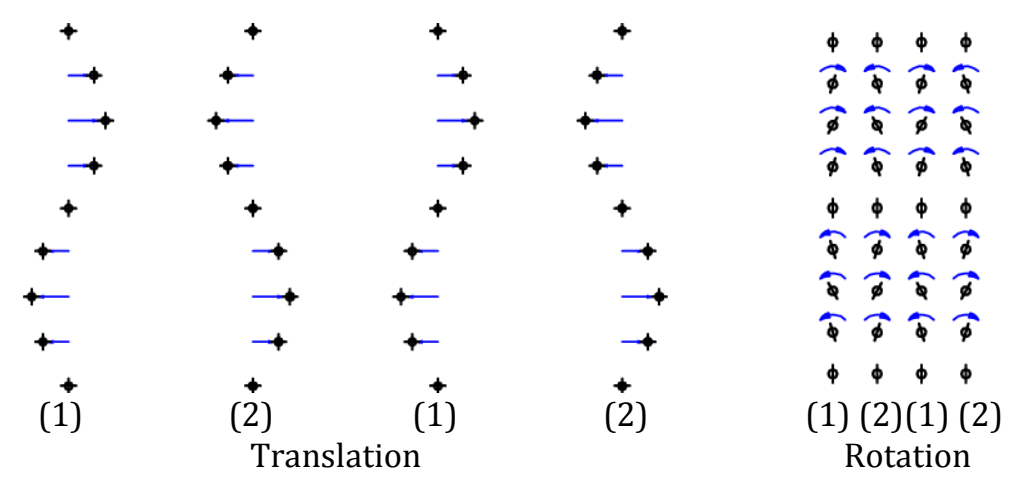

Figure 30. Discrete translation of rigid body periodically arranged on a straight line I.: two-phase translation

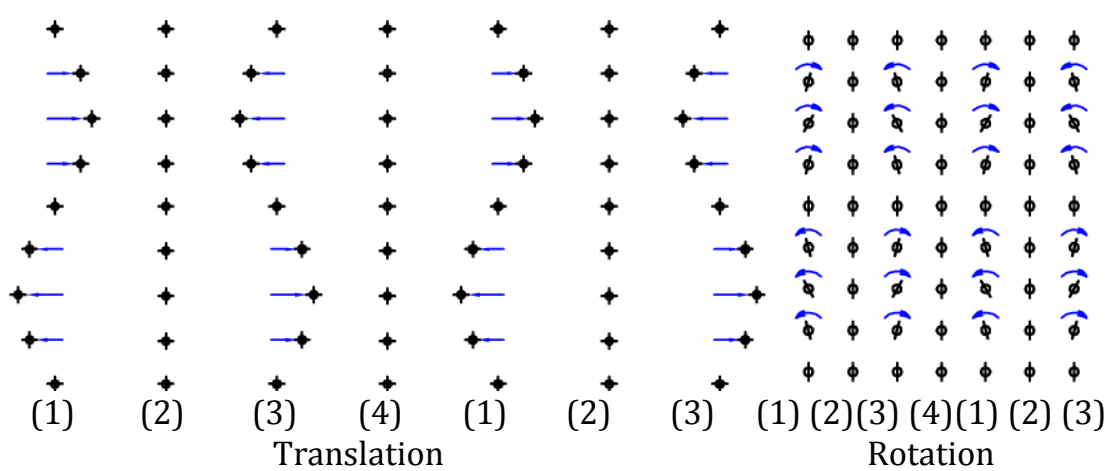

Figure 31. Discrete translation of rigid bodies periodically arranged on a straight line II.: three-phase translation

\section{Continuous system}

\subsection{The interpretation of the continuous system}

In the course of interpretation of continuous system we disregard the system of discrete elements, and the discrete system is modelled by a bounded and closed region of Euclidean space. "The elementary particle" of continuous body is the point in a geometrical sense. Formally the grid-mesh of lattice is kept but it will be used as a network (or coordinate) system (see Fig. 32.a). The kinematical degree of freedom of continuous body is the translation field. The continuous system will be named as continuous deformable solid body. 


\subsection{Disordered displacement (translation) field: the thermal motion}

The disordered translation cannot be interpreted because of the topological restriction of the points. Using physical observation the disordered (internal) translation can be brought into connection the homogeneous (temperature-dependent) change of volume (see Fig. 32.b).

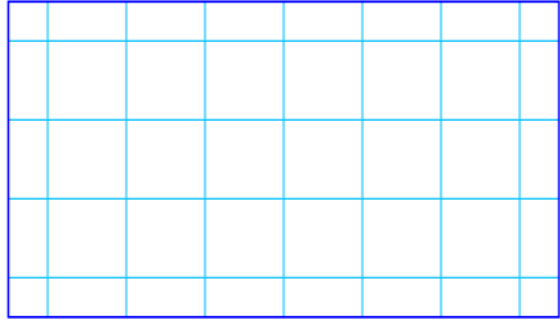

a) The beginning state of continuous system

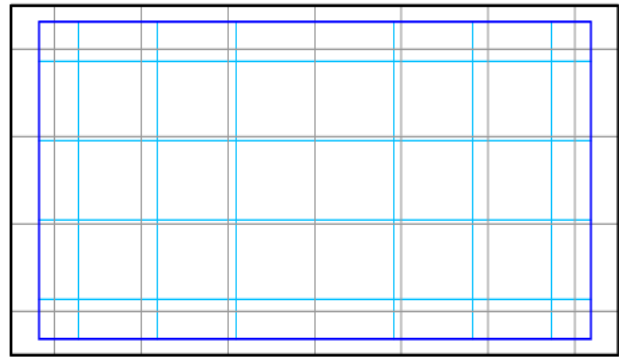

b) Homogeneous change of volume

Figure 32. Continuous system and the disordered movement

\subsection{Ordered displacement (translation) field}

Homogeneous local translation: global rigid body like translation.

Rotation around a point: global rigid body like rotation.

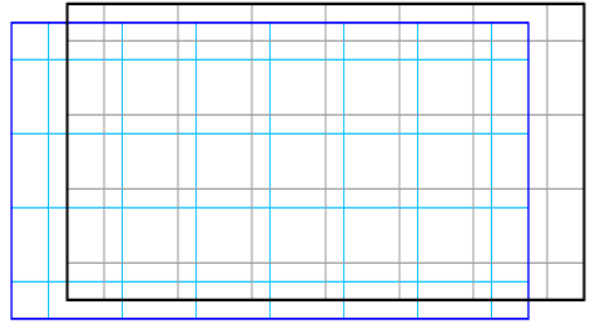

a) Translation

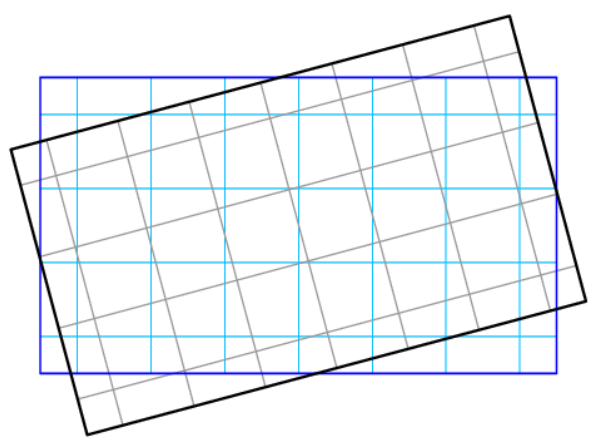

b) Rotation

Figure 33. The rigid body like translation and rotation of the continuous deformable solid body

Linearly varying in magnitude translation in direction of translation vector, or in direction orthogonal to translation vector: homogeneous elongation and homogeneous (simple) shear, respectively.

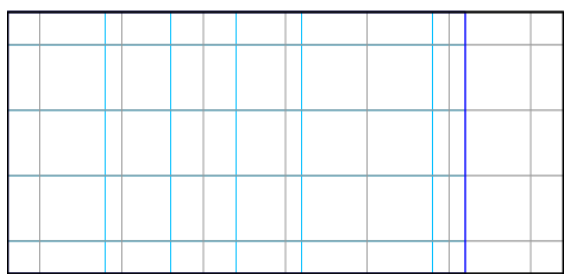

a) Homogeneous elongation

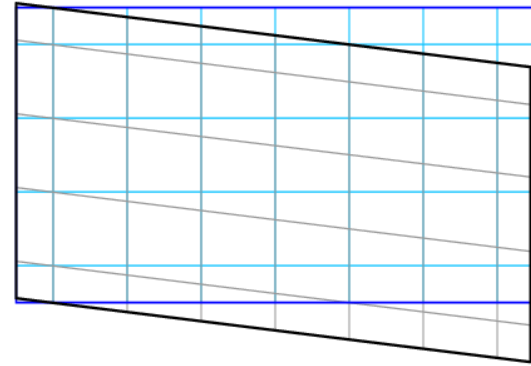

b) Homogeneous (simple) shear

Figure 34. Homogeneous elongation and homogeneous (simple) shear of the continuous deformable solid body 
Quadratically varying in magnitude translation in direction of translation vector, or in direction orthogonal to translation vector: linearly varying elongation and linearly varying (simple) shear.

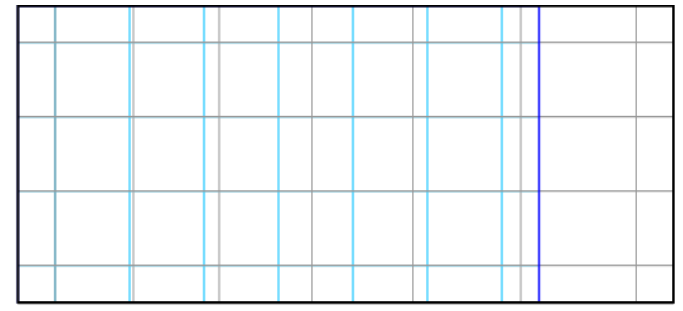

a) Linearly varying elongation

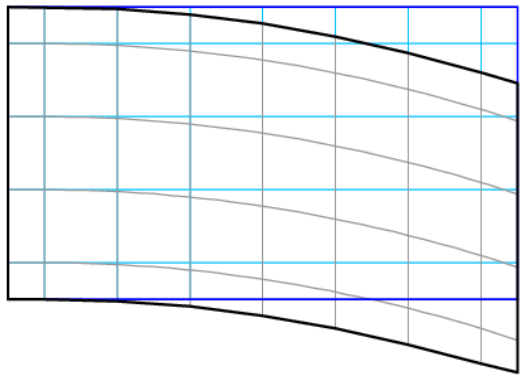

b) Linearly varying (simple) shear

Figure 35. Linearly varying elongation and linearly varying (simple) shear of the continuous deformable solid body

The geometrical points sitting on the favoured lines move in the direction orthogonal to favoured lines according to quadratic law and move in the direction of the favoured lines keeping (according to the one of the geometrical hypotheses of rod's and shell's theory) orthogonality to the favoured line: pure bending per track (Fig. 36.a), or in full cross section (Fig. 36.b).

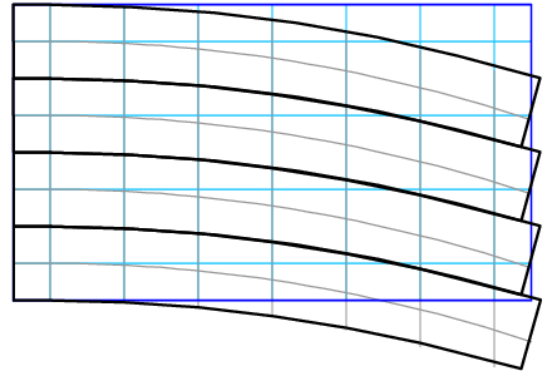

a) Pure bending per track

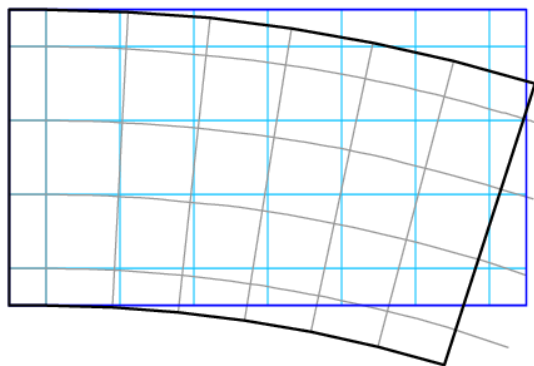

b) Pure bending in full cross section

Figure 36. Translation in the direction orthogonal to favoured lines according to quadratic law and in the direction of the favoured lines keeping orthogonality to the favoured line: pure bending per track, or in full cross section of the continuous deformable solid body

Notes. 1. Continuous model keeping orthogonality to the favoured line is analogue to the movement of system of material points SNRN, as the points have no directions (see Chapter 2.). 2. To interpret pure bending the existence of geometrical hypothesis is necessary and sufficient because of the rotation of geometrical point does not interpreted.

\section{Relationship of continuous and discrete systems}

The three systems are formally the same. To proves it enough to show the models of three systems and a general pictures on deformed states (see Fig. 37.). The representation convinces us that the three models ultimately describe the same. At the same time, there is a small "trick" or some "cheating" in the depiction. Because of the angle between the coordinate lines changes during the deformation, i.e. the representation of the model made of the rigid bodies does not correspond to reality (note the exclamation mark between the parentheses in the inscription Fig. 37.d). The axial crosses of the rigid bodies and the trihedron in the intersection point of the coordinate lines do not have to be coincide: the angles enclosed by the tangents of the deformed coordinate lines cannot be right angles, because of 
the body would have moved rigidly. Exceptions. 1. The case of existing of the geometrical hypotheses of rod's and shell's theory corresponding to the orthogonality (pure bending). 2. In the case of small deformation (irrespective of the magnitude of the translation) the angle change is small (see e.g. [10]), so the layout shown in Fig. 37.d approximates reality. Fig. 37.d must in fact be drawn up by showing the independent rotation of each rigid body. The axial cross positions resulting from the rotation of rigid bodies are shown in Fig. 38.a, while the shape of the grid lines connecting rigid bodies is illustrated in Fig. 38.b.

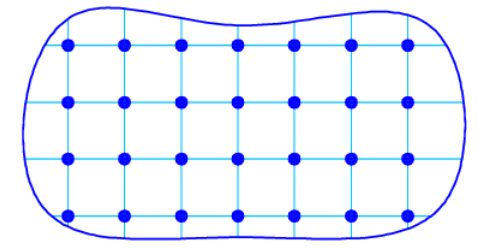

a) Modell built from material point

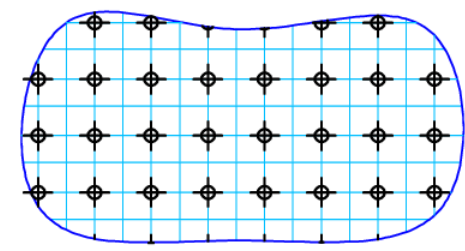

c) Model built from rigid body

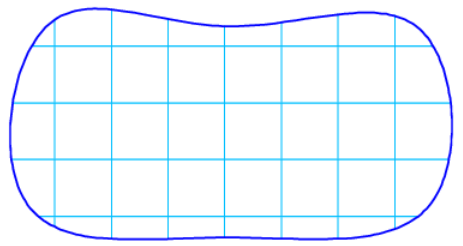

e) Continuous model

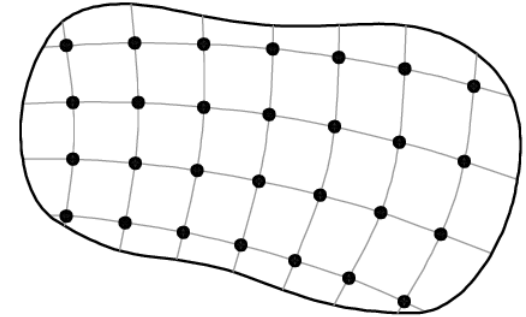

b) The deformations of the set of grid-mesh of lattice is continuous

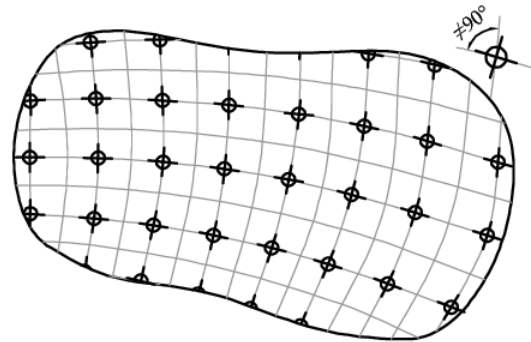

d) The deformations of the set of grid-mesh of lattice and the rotation of rigid body is continuous (!)

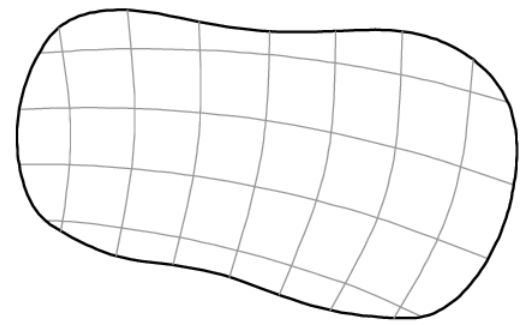

f) The deformations of set of coordinate lines is continuous

Figure 37. The three models and their continuous deformations

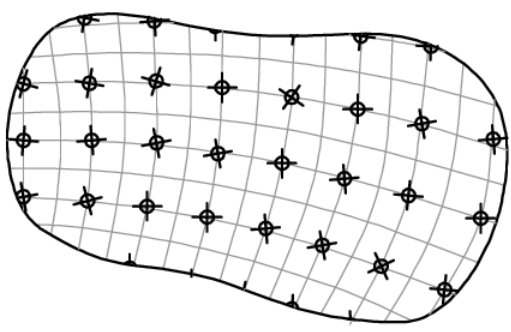

a) Rotation of rigid bodies

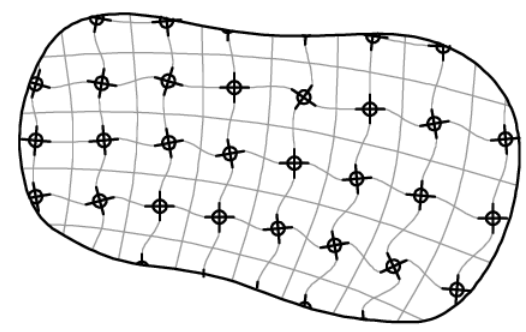

f) The deformations of set of mesh lines is continuous

Figure 38. Presentation of deformation of model built up rigid bodies with presentation of rotations of rigid bodies 
It can be seen from the Fig. 38. that, considering the rotations of rigid bodies, this model cannot be regarded as an equivalent model either to discrete model consisting of material points, or to continuous model (of geometrical points).

In addition to the above mentioned deviation there is a significant difference between the model consisting of rigid bodies and the other two models. Namely, the rigid bodies forming the deformable body can change their spatial situation and accordingly the shape of the grid lines connecting the rigid bodies will change, but the size and shape of the deformable body will not change (see Figure 39.).

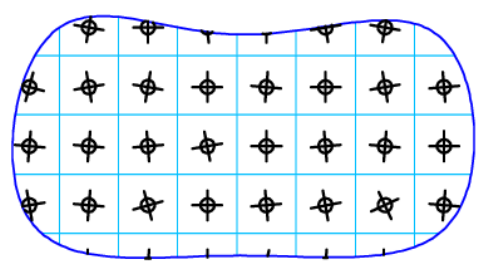

a) Rotations of rigid bodies

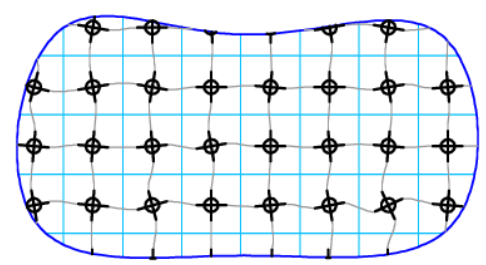

b) The deformations of set of mesh lines

Figure 39. Internal deformation of model consisting of rigid bodies

Note. Such behaviour among solid bodies is not known $[1,2,14]$.

\section{Summary}

The following substantive differences are found between the motion of continuous and of discrete system.

- In the discrete system continuous (collective) and discrete motion form can be interpreted, while in the continuous system only continuous (collective) motion form can be interpreted.

- In the discrete system the discrete motion form can be disordered (thermal motion) and ordered (patterned) (optical vibration in crystal).

- In the continuous system, as the real material system is discrete, disordered form of motion can be interpreted with change of volume (thermal expansion).

- The elements of discrete system can be modelled by material points as well as by rigid bodies. Accordingly to the choice, the kinematical degree of freedom of discrete system is either translation, or translation and rotation.

- The continuous system freeze the rotational kinematical degree of freedom of elements built the material. The elements of continuous system that is the geometrical points of Euclidean space have only translational kinematical degree of freedom, the rotational kinematical degree of freedom cannot be interpreted.

\section{References}

[1] C. Bach (1917) Elastizität und Festigkeit. Siebente Auflage. Springer, Berlin.

[2] J. Bell (1973) The Experimental Foundations of Solid Mechanics. In Mechanics of Solid I-IV. Vol. I. Springer, Berlin - Heidelberg, New York - Tokyo, 1984. (Originally published as Encyclopaedia of Physics VIa/I 1973.) 
[3] O. Bottema - B. Roth (1990) Theoretical kinematics. Dover, New York. (First Ed. 1979.)

[4] E. Cosserat - F. Cosserat (1909) Théorie des Corps Déformables Hermann et Fils. The Cornell University Library Digital Collections (Reprint 2017.)

[5] A. C. Eringen (1998) Microcontinuum Field Theories. Springer, New York, Berlin/Heidelberg.

[6] E. Kröner (ed.) (1968) Mechanics of Generalized Continua. Proceedings of the IUTAM-Symposium on the Generalized Cosserat Continuum and the Continuum Theory of Dislocations with Applications, Freudenstadt and Stuttgart (Germany) 1967. Springer-Verlag, Berlin - Heidelberg New York.

[7] I. A. Kunin (1975) Elastic Media with Microstructure I., II. Springer-Verlag, Berlin - Heidelberg New York, 2011-12 (translated from Russian, 1975.)

[8] G. Lámer (1984) Contradictions in the Theory of Micropolar Elasticity and Their Causes = Newsletter, Techn. Univ. of Budapest, II (1), 1984. pp. 12-16

[9] G. Lámer (2003) Solid and Soft Body with and without Structure. In: Quasi-Static Deformations of Particular Materials. Proceedings of QuaDMP'03 Workshop. 25-28 august 2003. Ed.: K. Bagi. P. Co. of BTU, Budapest. pp. 159-166.

[10] G. Lámer (1985) Notes on the Theory of Large Displacement with Small Strain. Periodica Politechnica. 29 (1-2) pp. 53-65

[11] G. Lámer (2018) Continuous and Discrete Models in the Mechanics of Deformable Solid Bodies. Proceedings of the Annual Session of Scientific Papers. Vol. XVII. (XXVII) "IMT Oradea" 2018. May 31. - June 1. pp. 125-130

[12] A. I. Lurie (1970) Theory of Elasticity. Springer. Berlin - Heidelberg - New York, 2005. (translated from Russian, 1970.)

[13] E. Németh (1963) Hydrodynamics. Tankönyvkiadó, Budapest. (In Hungary)

[14] W. N. Jr. Sharpe (ed.) (2008) Springer Handbook of Experimental Solid Mechanics. Springer, New York.

[15] C. Truesdell - R. A. Toupin (1960) The Classical Field Theories. In: Encyclopaedia of Physics. Ed. by S. Flügge. Vol III/1. Principles of Classical Mechanics and Field Theories. Springer, Berlin Göttingen - Heidelberg. pp. 226-793. 